\title{
Consensual Nature of Scientific Truths
}

\author{
Sergey Lebedev \\ Bauman Moscow State Technical University \\ Moscow, Russia 105005 \\ E-mail: saleb@rambler.ru
}

\begin{abstract}
The article enunciates and justifies the consensual conception of the scientific truths nature. The basis of this conception is a constructive and representational interpretation of scientific knowledge at all levels of scientific cognition: sensual, empirical, theoretical and meta-theoretical. We consider the consensual paradigm of the scientific truths nature as an alternative to two traditional paradigms in understanding the scientific truths nature: empiricist and rationalist conceptions. According to the first conception, the basis, source and criterion of truth of any scientific knowledge element is an empirical experience. However, the truth nature of mathematical knowledge and scientific theories in modern natural sciences is contrary to the empiricist conception of scientific truth. As follows from the rationalist conception, any scientific truth is a product of thinking, the main methods of which are intuition, idealization and deduction. But this conception contradicts the ways of obtaining and verifying the truth of sense-data in science along with science facts and empirical laws. Unlike experimentalism and rationalism the process of scientific cognition within the framework of consensual paradigm is thought of as having an intrinsically social character, and its real subject is neither a transcendental subject nor an individual scientist, but only the disciplinary scientific community. On the contrary, the process of obtaining, justification and estimation of the scientific cognition results rests in a substantial way not only on the subject-object cognitive relation, but also on the communicative relationships within the scientific community. In view of this, the recognition of any part of scientific knowledge by the scientific community as true (or untrue) always has an expert and consensual character.
\end{abstract}

Keywords-scientific cognition; scientific truth; the subject of scientific cognition; part of scientific knowledge; level of scientific knowledge; scientific consensus

\section{INTRODUCTION}

One of the distinctive features of scientific cognition unlike the other types of human cognition (artistic, philosophical, ordinary, religious) is its fundamentally objective character [1]. Therein lies not only the power of scientific cognition, but also its frontiers. It is obvious that by virtue of the scientific cognition orientation towards the cognition of objects, the substance of scientific knowledge significantly depends on the substance of cognizable objects. On the other hand, it is equally evident (and it is demonstrated by both the history of science and its contemporary state) that the relationship between the objects and the knowledge about them does not have a character of categorical determination on the part of the object. The empirical evidence that there is no such determination is a continuous availability in the science during all its history of many conflicting conceptions, models and theories of the same objects. This is because the cognitive relationship between the objects and the knowledge about them has a representative nature rather than a reflective one. The representation of the cognizable object in the consciousness depends not only on the object substance but also on the particular means of cognition applied by the subjects [2]. These means notably include: 1) the nature of cognitive and practical problems that the scientist solves; 2) using the specific segment of the previously accumulated scientific knowledge; 3) specific scientific language with its consistently concrete and restricted expressiveness; 4) a set of cognition methods acknowledged as legitimate in the science of the definite historic period ("ideals and norms of scientific investigation"); 5) creative potential of scientists as the subjects of scientific cognition [3]. So, the representation of the cognizable objects in the scientific consciousness and in the scientific knowledge always has a double determination: 1) on the part of the objects and their properties and 2) on the part of the scientific cognition subjects always using a certain variety of the cognizable object representation means. Account of the scientific cognition representational nature and double determination of the scientific knowledge substance allows drawing the important philosophical conclusion that the scientific knowledge upon the whole as well as its any segment always has a subject-object character [4]. The assertion of this statement has a crucial significance for the correct understanding of the scientific truth nature and recognition of its consensual character [5]. The evidence for the representational and constructive character of the scientific cognition is not only the existence of its many cultural and historical types and states in the real scientific history, but also of a number of fundamentally different in their methods fields of scientific knowledge (logic, mathematics, natural science, social and humanitarian sciences, engineering sciences, interdisciplinary research). The criteria of the scientific rationality for different fields of scientific knowledge have not only the common content, but also the essential difference [6]. When underlying rationale for the consensual nature of scientific knowledge it is of paramount importance to realize the qualitative difference in ontological and methodological relation between the basic levels of scientific knowledge [7]. 


\section{Consensual Character of the Sensual SCIENTIFIC KNOWLEDGE TRUTHS}

In any developed science there are four basic levels of scientific cognition and respective types of knowledge: 1) sensual; 2) empirical; 3) theoretical and 4) meta-theoretical. The sensual level of the objects cognition is underlying in any science. The main results of the sensual level of cognition in the science are the data of sensorial perception and experiment. The means of obtaining the scientific knowledge of sensual level include: naturalistic observations, experiment (material impact upon the object of cognition) and the devices used for fixing the results of the objects sensual cognition. Let us list the primary factors influencing both on constructing and estimating the truth of the sensual cognition results: 1) the choice of the research subject area (consensual factor); 2) the substance (properties) of cognized objects (objective factor); 3) consensus of scientific community's opinion on the selection and correctness of using certain means of sensual cognition. For example, these means can be obtaining sensual information about the object of cognition only on the basis of naturalistic observation, without the material impact upon it (pedagogics, psychoanalysis, linguistics, cosmology, sociology, etc.). But it can also be obtaining sensual information about the object of cognition, though without the material impact upon it, but with the aid of using devices as the amplifiers of the sensual cognition organs. Finally, it can also be specific (but always quantitatively fixed) material impact upon the object of cognition in the course of the experiment. It is well known that particularly this method is the main means of obtaining sensual information about the objects of cognition in natural and engineering sciences. The overall conclusion is that even the sensual level of cognition in science and its results have a substantively consensual nature due to the fact that they are based on the recognition or non-recognition of the legitimacy of the particular set of tools which is used at this level for the representation of the cognized object's substance [8].

\section{CONSENSUAL NATURE OF THE EMPIRICAL KNOWLEDGE IN SCIENCE}

Let us call the primary structural units of the empirical knowledge in science: 1) observation protocols; 2) science facts as the statistical generalizations of the protocols; 3 ) different types of empirical laws (deterministic, probabilistic, causative, functional, structural); 4) phenomenological theories (interconnected system of empirical laws of a certain subject area) [9]. The means of the objects representation at the empirical level of the scientific cognition are: 1) the description of the observation results in the natural (ordinary) language or in the artificial (engineering) language (instrumental language comprising the names of the devices in use, the description of the instrument operations, the names of the measured values and the used systems of physical values); 2) the applied methods of constructing the empirical knowledge: abstracting, generalization, definition, different kinds of induction, classification etc.; 4) the applied methods of checking and justifying the empirical knowledge (empirical verification, confirmation, prediction, refutation, logical systematization etc.). The factors determining the consensual character of the empirical knowledge truth include: 1) creative construction by the scientists of the abstract objects as the straightforward subject-matter of the empirical level of knowledge and their acceptance by the scientific community as objective and significant for the science; 2) evaluation by the scientific community of the appropriateness and efficiency of using the particular methods of empirical cognition; 3) consensus of the disciplinary community opinion on the truth and proof of empirical laws and theories [10].

\section{Consensual Nature of The TheORETICAL LEVEL OF KNOWLEDGE TRUTHS IN SCIENCE}

The main procedures and methods of the theoretical level of scientific cognition are: 1) constructing the initial and derivative ideal objects of theory; 2) the introduction and description of the theoretical objects equations of state; 3 ) the construction of theory as the logically probative system of knowledge about the ideal objects on the basis of specific theoretical hypotheses and principles; 4) the acceptance of the determined system of logic with its deduction rules; 5) finding the empirical interpretation of the theory; 6) the description of potential areas for the practicality of theory [11]. These are the main consensual factors influencing on the acceptance and estimation of the theoretical knowledge truth: 1) estimation by the scientific community of the legitimacy of the initial and derivative scientific theory objects; 2) estimation by the scientific community of the legitimacy and efficiency of the applied means and methods of theoretical cognition; 3) consensus among the members of disciplinary scientific community regarding the truth of prime statements and principles of theory; 5) expert assessment by the disciplinary scientific community of the relevance, practical significance and efficiency of the particular theory [12]. The overall conclusion is that the assessment of the truth and proof of both the individual elements of scientific theory and theory on the whole also has notably consensual character.

\section{Consensual Character of THE Meta- THEORETICAL LEVEL OF SCIENTIFIC KNOWLEDGE TRUTHS IN SCIENCE}

Meta-theoretical level of scientific cognition and knowledge is the most general. The main types of metatheoretical knowledge in science are: 1) fundamental (paradigm) scientific theories; 2) general scientific knowledge (scientific global picture and general scientific methodology); 3) philosophical foundations of science of various contents (ontological, gnosiological, axiological, sociocultural). The main procedures of scientific cognition at the meta-theoretical level are: 1) evaluating scientific theories with regard to their correspondence to general and sectorial criteria of scientific rationality; 2) assessment of scientific theories in the matter of their logical proof, empirical justifiability, practical utility; 3) estimating scientific theories concerning their correspondence to paradigm theories of a particular area of knowledge; 4) reconstruction of the philosophical underpinnings of scientific theory; 5) analyzing the benefits and drawbacks of 
a specific theory in comparison with the other similar theories in the same area of knowledge. The consensual factors of assessing the truth of the meta-theoretical knowledge in science include: 1) selection by the scientific community of the specific scientific theories as the objects of meta-theoretical cognition; 2) selection by the scientific community of certain fundamental theories or philosophical underpinnings of science; 3) formulation and acceptance by scientists of certain conceptions regarding scientific rationality as well as specific methodological requirements to constructing and justifying scientific theories; 4) assessment and selection of some scientific theory as the best among the other similar theories; 5) preference by the scientific community of one metatheories or philosophical underpinnings of science to the other amidst the constantly taking place pluralism in the field of metatheories and philosophical conceptions [13]. It is obvious that the acknowledgment of the truth of one or another fragment of meta-theoretical knowledge in science has a clearly consensual nature.

\section{CONCLUSION}

The estimation of the scientific knowledge truth at each level has a significantly consensual character. This refers to all the areas of science and to all levels of scientific knowledge: sensitive, empirical, theoretical and metatheoretical level. Understanding the consensual character of the scientific knowledge truth allows generating more sensible beliefs about the regularities of scientific cognition functioning and development compared to not only positivistic but also postmodern conceptions and scientific cognition models.

\section{REFERENCES}

[1] Lebedev S.A. Methodology of scientific cognition. Monograph. Moscow, Prospekt Publ., 2015, 256 p.

[2] Inozemtsev V. A., IvlevV.Yu., Ivleva M.L. Artificial Intelligence and the Problem of Computer Representation of Knowledge.Proceedings of the 2nd International Conference on Contemporary Education, Social Sciences and Humanities (ICCESSH 2017).Part of series Advances in Social Science, Education and Humanities Research.Moscow, Russia, 2017. V.124,pp.1151-1157.

[3] Lebedev S.A. Ideals and norms of scientific research and their methodological function. Humanities Bulletin of BMSTU, 2018, no. 3, pp. 521-525.

[4] Gubanov N.I., Gubanov N.N. The role of mentality in the development of society: sociocultural hypothesis // Vestnikslavianskikh kultur-bulletin of slavic cultures-scientific and informational journal. 2017. Vol. 43. № 1. P. 38-51.

[5] Lebedev S.A. The nature of truth in science. Humanities Bulletin of BMSTU, 2017, no. 12(62), pp. 1-26

[6] Lebedev S.A. The Structure of Scientific Rationality // Voprosy filosofii. 2017. № 5. P. 66-79.

[7] Lebedev S.A. The Reassembly of the Epistemology // Voprosy filosofii. 2015. № 6. P. 53-64.

[8] Lipkin A.I., Pronskikh V.S. Theoretical components in the experiments on the elementary particle accelerators. RUDN Journal of Philosophy, 2010, no. 3, pp. 56-65.

[9] Gubanov N.I., Gubanov N.N. Course of lectures in philosophy of science // Herald of the Russian Academy of Sciences. 2015. V. 85. №10. P. 946-948. DOI: 10.7868/S0869587315100059.
[10] Lebedev S.A., Koskov S.N. Conventions and consensus in the context of contemporary philosophy of science. Novoe $\mathrm{v}$ psikhologopedagogicheskikhissledovaniyakh(The new in psychological and pedagogical research), 2014, no. 1, pp. 7-13.

[11] Oseledchik M.B., IvlevV.Yu., Ivleva M.L. A new paradigm for analyzing knowledge transfer processes. Proceedings of 4th International Conference on Education, Language, Art and Intercultural Communication (ICELAIC 2017)Part of the series ASSEHR. Moscow, Russia, pp. 766-770.

[12] Nekhamkin V.A. A counterfactual Challenge of the Past: Ways of Negotiation // Herald of the Russian Academy of Sciences. 2017. Vol 87. Issue 2. P. 191-198. DOI: 10.1134/S1019331617020046.

[13] Lebedev S.A. The main paradigms of epistemology and the philosophy of science // Voprosy filosofii. 2014. № 1. P. 72-82. 